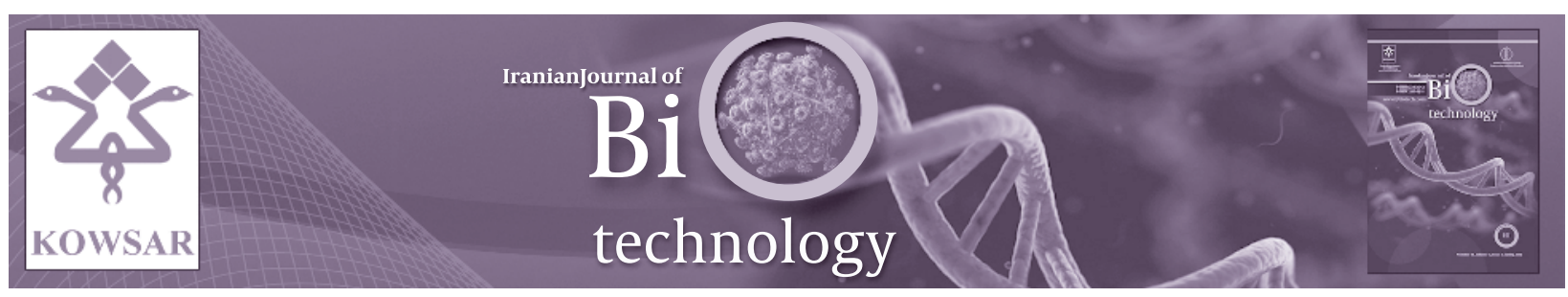

\title{
Molecular Cloning and Characterization of the Phenylalanine Amino- mutase Gene From Taxus baccata L.
}

\author{
Abolghasem Abbasi Kajani ${ }^{1}$, Mohamma Reza Mofid ${ }^{2,{ }^{*}}$, Khalil Alami Saeid $^{3}$ \\ ${ }^{1}$ Department of Biotechnology, Faculty of Advanced Sciences and Technologies, University of Isfahan, Isfahan, IR Iran \\ 2 Department of Biochemistry, School of Pharmacy and Pharmaceutical Sciences, Bioinformatics Research Center, Isfahan University of Medical Sciences, \\ Isfahan, IR Iran \\ ${ }^{3}$ Ramin Agricultural and Natural Resources University, Mollasani, Ahwaz, IR Iran \\ *Corresponding author: Mohammad Reza Mofid, Department of Biochemistry, School of Pharmacy and Pharmaceutical Sciences, Bioinformatics Research \\ Center, Isfahan University of Medical Sciences, Isfahan, IR Iran, Tel: +98-3117922597, Fax: +98-3116680011, E-mail: mofid@pharm.mui.ac.ir.
}

\section{A B S T R A C T}

Background: Taxol is one of the most important anti-cancer drugs, which is obtained from yew trees (Taxus sp.). T+he first step in side chain assembly of taxol is catalyzed by phenylalanine aminomutase, which converts $\alpha$-phenylalanine to $\beta$-phenylalanine.

Objectives: In this study, for the first time, we report on the cloning, preliminary expression and characterization of a full-length gene and cDNA encoding phenylalanine aminomutase from Taxus baccata $\mathrm{L}$.

Materials and Methods: Comparison of the full-length gene with other ones identified from the Taxus species showed high similarity, particularly with Taxus $x$ media.

Results: The results showed that the expression level of this gene in Taxus baccata is very low and therefore this enzymatic step could be a rate limiting step in the taxol biosynthesis pathway. Successful amplification of the cDNA was only obtained from RNA samples isolated from methyl jasmonate elicited suspension cells of Taxus baccata. The cloned cDNA contained a $2064 \mathrm{bp}$ open reading frame encoding a protein composed of 687 amino acids. Sequence comparison analysis revealed that the gene is very similar $(98-99 \%)$ with respect to the nucleotide and amino acid sequences in different Taxus species and also share the signature active site motif (175ASG177).

Conclusions: The predicted structure of TbPAM was analyzed using bioinformatic tools. The results indicated that the protein has similar overall folding to tyrosine aminomutase.

Keywords: Molecular Cloning; Phenylalanine Aminomutase; Taxol; Taxus baccata L.

Copyright ( 2013, National Institute of Genetic Engineering and Biotechnology; Published by Kowsar Corp.

Article type: Research Article; Received: 11 Aug 2012; Revised: 30 Dec 2012; Accepted: 04 Mar 2013; Epub: 12 May 2013, Ppub: Spring 2013

Implication for health policy/practice/research/medical education:

This article has implication on researchers and scientific collaborators.

PPlease cite this paper as:

Abbasi Kajani A, Mofid MR, Alami Saeid K. Molecular Cloning and Characterization of the Phenylalanine Aminomutase Gene From Taxus baccata L. Iran J Biotech. 2013:11(2): 96-103. DOI: 10.5812/ijb.10714

Copyright (C) 2013, National Institute of Genetic Engineering and Biotechnology; Published by Kowsar Corp.

This is an Open Access article distributed under the terms of the Creative Commons Attribution License (http://creativecommons.org/licenses/by/3.0), which permits unrestricted use, distribution, and reproduction in any medium, provided the original work is properly cited. 


\section{Background}

Taxus species are known to produce a wide range of natural diterpenoids known as taxoids (taxanes), with approximately 350 identified forms. One of the bestknown taxoids, taxol, has been well proven to be a potent anticancer drug with powerful effects against a range of cancers. The production of taxol from the original plant source is very limited due to the low abundance and slow growth of Taxus trees and the low concentration of taxol in the trees (1). On the other hand, demand for the drug has increased considerably due to the expansion of clinical trials and treatments (2). Therefore, it seems that the only way to increase the yields of taxol and other clinically important taxoids by genotypes, is to study and manipulate the biochemical and enzymatic reactions in the taxoid pathway (3). This is a precondition for the eventual over expression of the slow steps and/or silencing of the subsidiary steps of the pathway (3). Generally, the taxol molecule consists of two components (Figure 1): the taxane ring moiety (10-deacetyl baccatin III) derived from the terpenoid pathway and C-13 side chain derived from the phenylpropanoid pathway (4). Since, the taxane ring moiety is much more readily available than taxol itself (4), the low content of taxol may be related to its side chain assembly. The taxol biosynthesis pathway contains about 20 distinct enzymatic steps (2). Tremendous progress has been made in the cloning and functional expression of the genes involved in the pathway. The first step in the side chain assembly of taxol is catalyzed by phenylalanine aminomutase (PAM) which converts $\alpha$-phenylalanine to $\beta$-phenylalanine. The activity of the enzyme was demonstrated in the soluble fraction of crude cell-free extracts of T. brevifolia (4). Feeding studies showed that $\beta$-phenylalanine is incorporated into both phenylisoserine and benzoate moieties of the side chain (4). Thus, PAM may limit the rate of taxol production at the commercial scale and could be an important target for genetic engineering in yew and its derived cell cultures to increase the taxol productivity. On the other hand, characterization of the enzyme is essential to understand the nature of taxol side chain biosynthesis (5). PAM gene from Taxus cuspidata and Taxus chinensis has been cloned and functionally expressed in Escherichia coli $(4,5)$. Until now, there is no report on the cloning of PAM gene from $T$. baccata. This species contains comparatively lower content of taxol and higher content of baccatin III in its needles than the other Taxus species (6).

Figure 1. Taxol Biosynthesis Pathway

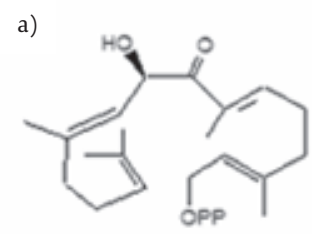

Geranylgeranyl diphosphate

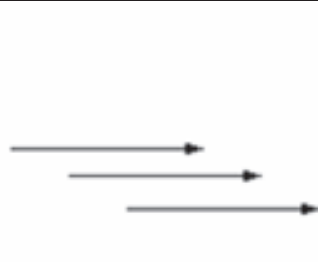

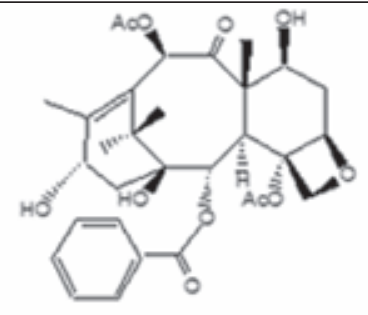

Baccatin III<smiles>N[C@@H](CC(O)O)c1ccccc1</smiles>

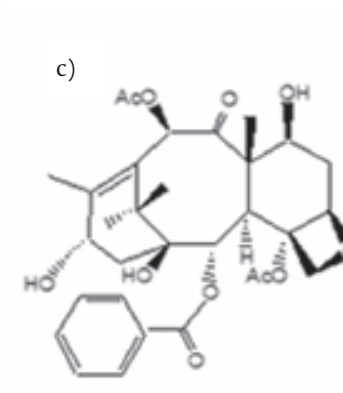

Baccatin III

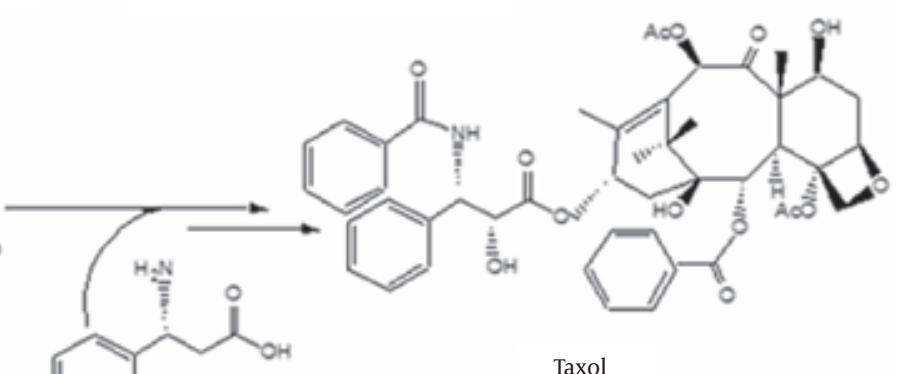

Taxol

a) biosynthesis of taxane ring moiety from the terpenoid pathway, b) conversion of $\alpha$-phenylalanine to $\beta$-phenylalanine by phenylalanine aminomutase (PAM), c) attachment of C-13 side chain to the Baccatin III and biosynthesis of Taxol. 


\section{Objectives}

In this paper, we described the cloning and characterization of the PAM gene from T. baccata (TbPAM) as an initial step to investigate the physiological role of the gene in this species.

\section{Materials and Methods}

\subsection{Materials}

General molecular biology reagents were obtained from Fermentas (Germany), Merck (Germany) or Sigma (St. Louis, MO), unless otherwise indicated. Enzymes for PCR and RT-PCR were purchased from Roche (Roche Applied Science, Germany). Cloning of the full-length gene and cDNA were carried out using pTZ57R/T vector (InsTAclone ${ }^{\mathrm{TM}}$ PCR Cloning Kit) from Fermentas (Germany). Plant growth regulators, nutrients and other plant tissue culture materials were purchased from Duchefa (Biochemie B.V., Netherlands).

\subsubsection{Plant Material, Media and Culture Conditions}

Young leaves and stems were collected from adult Taxus baccata trees at the flower garden of Isfahan in Isfahan, Iran. Callus was obtained from the leaf explants in B5 medium containing 2 mg. $\mathrm{L}^{-1} \mathrm{NAA}$ and $0.2 \mathrm{mg} . \mathrm{L}^{-1}$ kinetin in dark condition as previously described (7). In order to prepare cell suspension culture of Taxus, three grams of two-month old callus were added to $50 \mathrm{~mL}$ of the same liquid B5 medium in a $250 \mathrm{~mL}$ flask. The flask was incubated for 21 days in darkness at $25^{\circ} \mathrm{C}$, shaking at $110 \mathrm{rpm}$. The culture was elicited at day 14 by methyl jasmonate with a final concentration of $100 \mu \mathrm{M}$. The elicited cells were separated from the remaining medium at day 21 by centrifugation at $5000 \mathrm{rpm}$ for $10 \mathrm{~min}$ and used for RNA isolation procedure.

\subsection{DNA and RNA Isolation}

Total RNA was extracted from the elicited cell suspension using a special method for Taxus species (3). Briefly, the cells were ground to a fine powder in liquid nitrogen and added to $10 \mathrm{~mL}$ of preheated extraction buffer containing a high concentration of $\beta$-mercaptoethanol (4\%) and PVP (4\%). The homogenate was then extracted with chloroform: isoamylalcohol $(24: 1 \mathrm{v} / \mathrm{v}), 3$ times. The upper phase was carefully mixed with a 0.5 volume of $3 \mathrm{M}$ sodium acetate $(\mathrm{pH} 4.8)$, incubated at $-20^{\circ} \mathrm{C}$ for $30 \mathrm{~min}$ and centrifuged. The supernatant was mixed gently with $1 / 4$ volume of $10 \mathrm{M} \mathrm{LiCl}$ and placed at $4^{\circ} \mathrm{C}$ overnight. The RNA was pelleted by centrifugation, dissolved in $100 \mu \mathrm{L}$ DEPCtreated water, and then treated with RNase-free DNase I (Fermentas). The homogenate was extracted twice with chloroform: isoamylalcohol and the RNA was pelleted using ice-cold absolute ethanol, and finally dissolved in DEPC treated water. Genomic DNA was extracted using a CTAB (hexadecyltrimethylammoniumbromide)-based method (3) and used for cloning of the full length PAM gene. The quality and concentration of RNA and DNA samples were examined by agarose gel electrophoresis and spectrophotometer (ND-1000, USA) analysis.

\subsection{PCR and RT-PCR}

The PCR was done in a total volume of $25 \mu \mathrm{L}$ containing 2 $\mathrm{mM} \mathrm{MgCl} 2,10$ pmol of each primer of PAM (Forward primer: 5'-TTTTGAATTCATGGGGTTTGCCGTGGAATC-3' ; reverse primer: $5^{\prime}$-TTTTGGTACCCTAGACGCCGTTGGCGCA-3'), 200 $\mu \mathrm{M}$ of each dNTPs, 1 unit Taq DNA polymerase (Roche Applied Science, Germany), and 100 ng of genomic DNA in $1 \mathrm{x}$ PCR buffer. The PCR amplifications were carried out using a MyCycleTM Thermal cycler (Bio-Rad, USA). Each PCR was initiated by a preheating step for $3 \mathrm{~min}$ at $94^{\circ} \mathrm{C}$ followed by 30 cycles consisting of a denaturation step $\left(30 \mathrm{sec}\right.$ at $\left.94^{\circ} \mathrm{C}\right)$, primer-annealing step $\left(30 \mathrm{sec}\right.$ at $61^{\circ} \mathrm{C}$ ), and elongation step ( $3 \mathrm{~min}$ at $72^{\circ} \mathrm{C}$ ). The final elongation step was extended for $8 \mathrm{~min}$. The first-strand cDNA synthesis was done using AMV reverse transcriptase with Oligo-(dT)17 primer and approximately $2 \mu \mathrm{g}$ of total RNA according to the manufacturer's instructions (Roche Applied Science, Germany). The second strand amplification was carried outusing high fidelity PCR system (Roche Applied Science, Germany) and the PCR conditions similar to that explained before. The PCR products were then analyzed by gel electrophoresis.

\subsection{Cloning of Full-Length cDNA and Genomic Gene}

The PCR product of appropriate size was gel purified and extracted carefully using a DNA Extraction Kit (Fermentas). The purified fragment was then cloned into the pTZ57R/T vector according to the manufacturer's instructions (InsTAclone ${ }^{\mathrm{TM}}$ PCR Cloning Kit, Fermentas). The vectors were introduced into E. coli XL1-Blue cells by electroporation with a Gene Pulser XcellTM apparatus (Bio-Rad, USA) and the cells were cultured overnight at $37^{\circ} \mathrm{C}$ in Luria Bertani (LB) medium containing $50 \mu \mathrm{g} \cdot \mathrm{mL}^{-1}$ ampicillin. The positive clones were selected based on lacZ gene marker and finally sent for sequencing to the Macrogene Company (Seoul, Korea).

\subsection{Preliminary expression and Analysis of Recom- binant Protein}

The recombinant plasmid containing PAM cDNA was cotransformated into E. coli BL21(ìDE3) cells (Merck Biosciences, Schwalbach, Germany) and cultivated at $30^{\circ} \mathrm{C}$ overnight in $5 \mathrm{ml} \mathrm{LB}$ medium containing $100 \mu \mathrm{g} \cdot \mathrm{mL}^{-1}$ of ampicillin. The overnight culture was then inoculated into $500 \mathrm{~mL}$ of the same medium and cultivated at $30^{\circ} \mathrm{C}$. The culture was grown at $30^{\circ} \mathrm{C}$ to $\mathrm{A} 600=0.5-0.6$, and then induced by addition of $0.5 \mathrm{mM}$ isopropyl $\beta$-D-thiogalactopyranoside (IPTG) to express the PAM gene. The cells were grown for another $4 \mathrm{~h}$ at $30^{\circ} \mathrm{C}$ and finally the presence of the respec- 
tive protein was detected using SDS-PAGE (12\% Laemmli gels). Protein concentrations were determined on the basis of the calculated extinction coefficient at $280 \mathrm{~nm}: 51185$ $\mathrm{M}-1 \mathrm{~cm}^{-1}$.

\subsection{Bioinformatics Analysis}

BLAST was used to investigate for homology in the GenBank database and the Gene Runner program was used to deduce the amino acid sequence of the gene. Multiple sequence alignments were performed using the DNASTAR Lasergene core suit software. Analysis and characterization of the protein were performed using protparam belonging to the Swiss Institute of Bioinformatics [http:/ www.expasy.ch/tools/protparam.html/]. Secondary structure of TbPAM was analyzed by the Swiss-pdb viewer tool (version 4.01). Tertiary structure of TbPAM was predicted by using phyre webserver (Version 0.2) and Swiss Model 8.05 [http://www.expasy.org/swissmodel/].

\section{Results}

\subsection{DNA and RNA Isolation}

Isolating high-quality samples of nucleic acid is a critical step in many molecular biology experiments especially in cDNA synthesis of low expressed genes or rare mRNAs. Degradation and contamination of the RNA samples during the isolation process may cause the failure of the following experiments. Different tissues of Taxus contain high levels of secondary metabolites especially phenolic compounds that are rapidly oxidized and vigorously bond nucleic acids during isolation and finally result in brown and insoluble nucleic acid pellets. The presence of such powerful oxidizing agents in the samples may result in decreased purity and productivity of the final extracted nucleic acids. Agarose gel electrophoresis of nucleic acids also showed good quality of DNA and RNA samples with no considerable polysaccharide, polyphenol and protein contaminations (Figure 2). The results indicate that high quality DNA and RNA samples with appropriate yields can be obtained using the protocol.

\subsection{Cloning and Sequence Analysis of the Full- Length Gene}

For identification and amplification of the full length of thePAM gene from Taxus baccata, forward and reverse primers were designed based on highly conserved 5' and 3 ' terminal sequences of ORF regions of the PAM genes in other Taxus species. Digestion sites of EcoRI (under lined sequence) and KpnI (bold sequence) restriction enzymes were also added to the ends of primers for cloning the gene in the vector (pASK-IBA43plus) for subsequent experiments. In order to improve the digestion efficiency, four thymine nucleotides were also added to the 5'-ends of primers. As a result of these nonspecific nucleotides in primers, the annealing temperature was reduced considerably. PCR reaction using the primers and Taxus baccata genomic DNA (as template) resulted in the specific amplification of a single fragment of about $2250 \mathrm{bp}$ (Figure 2) that was cloned into the vector and sequenced subsequently. Investigating the sequence of the cloned fragment in Gene Bank showed high similarity to the sequences of PAM genes identified in other Taxus species. Moreover, specific amplification of the fragment in the PCR reaction confirmed the assumption that the 3'- and 5'ends of PAM gene from Taxus baccataare similar to those reported previously from other Taxus species. The cloned full-length of PAM gene from Taxusbaccata was $2233 \mathrm{bp}$ in lengh (designated as TbPAM, Genbank accession number: GU214709) and contained an exon within the gene from nucleotide 1092 to 1260 . Searches against Gen Bank using the BLAST algorithm revealed that the similarity between TbPAM and the gene obtained from Taxus $x$ media is more than that from Taxus canadensis and Taxus chinensis with respect to the sequence, length and exon site. Since Taxus $x$ media originated from the hybridization of T. baccata and T. cuspidate (8), such a result was predictable.

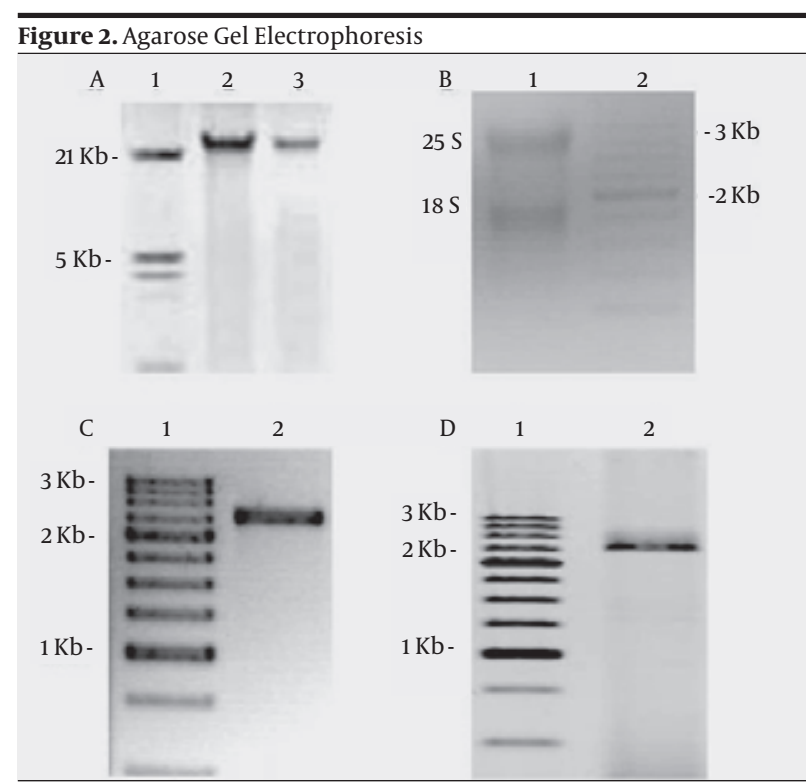

A, Taxus baccata Genomic DNA (1: Marker and 2, 3: Isolated DNA); B, RNA isolated from Taxus baccata (1:1 $\mu \mathrm{g}$ total RNA and 2: Marker); C, PCR product (1: Marker and 2: Full length of Phenylalanine aminomutase gene (2233 bp)); D, RT-PCR product (1: Marker and 2: Phenylalanine aminomutase cDNA (2064 bp)).

\subsection{Cloning and Sequence Analysis of the TbPAM} cDNA

Isolating RNA samples from the native tissues and also non-elicited cell suspensions of Taxus baccata to use in RTPCR reactions for synthesis of PAMcDNA, didn't result in any amplified product and all of the experiments failed. This may be related to the low expression level of the gene in Taxus baccata L. Moreover, the lower productivity of taxol 
in Taxus baccata compared to the other taxus species may also be related to the low expression of the PAMgene in this species and perhaps PAM would be a rate limiting enzyme in taxol biosynthesis pathway. However, more studies on the taxol biosynthesis pathway and the functions of the enzyme in the pathway could be useful in this context. Several studies have previously emphasized on the elicitor effects of methyl jasmonate on taxol synthesis from culture suspension of Taxus cells (9). Wang et al. (9) presumed that methyl jasmonate may activate the biosynthesis pathway from baccatin III to taxol and inhibit biosynthesis from baccatin III to cephalomannine. Based on this assumption, it seemed that methyl jasmonate is a suitable choice to induce the expression of PAM gene and consequently to increase the probability of gene amplification. Therefore, we isolated RNA samples from $T$. baccata cell suspensions, which were induced to result maximal taxol synthesis with $100 \mu \mathrm{M}$ methyl jasmonate at day 14 of culture. RT-PCR reactions by using these RNA samples resulted in amplification of a single fragment with lengh of about $2050 \mathrm{bp}$ (Figure 2). Sequencing result of the fragment (Figure 3 ) coincided with the sequences from ORF region of PAMcDNAs identified previously from other Taxus species (AY582743, AY724735). The cloned cDNA of TbPAM (Genbank accession number: GU214708) was 2064 bp in length. Nucleotide BLAST of the sequence from the NCBI website indicated that the gene shows only very minor sequence differences (almost as silent mutation) with the other known sequences of Taxus PAM (99\% similarity with Taxus chinensis and Taxus x mediaand $98 \%$ with Taxus canadensis). Since all Taxus species are known to be very closely related and are considered to represent geographic variants of the basic species, $T$. baccata such a result had been anticipated. The protein contained 687 amino acids (Genbank accession number: ADA57703) with a molecular weight of about $75.25 \mathrm{kDa}$ and an isoelectric point of 5.7. As also showen by Figure 4, amino acid sequence of TbPAM had high similarity (98\%) with the other known Taxus Phenylalanine aminomutases (AAU01184, AAU01183, and AAU01185). The minor differences are likely attributable to the species differences or to allelic variations. The secondary structure of TbPAM was analyzed by Swiss-pdb viewer tool (version 4.01) and the results showed that the putative TbPAM peptide contained 51\% alpha helix, $7 \%$ beta sheet, and $42 \%$ random coil

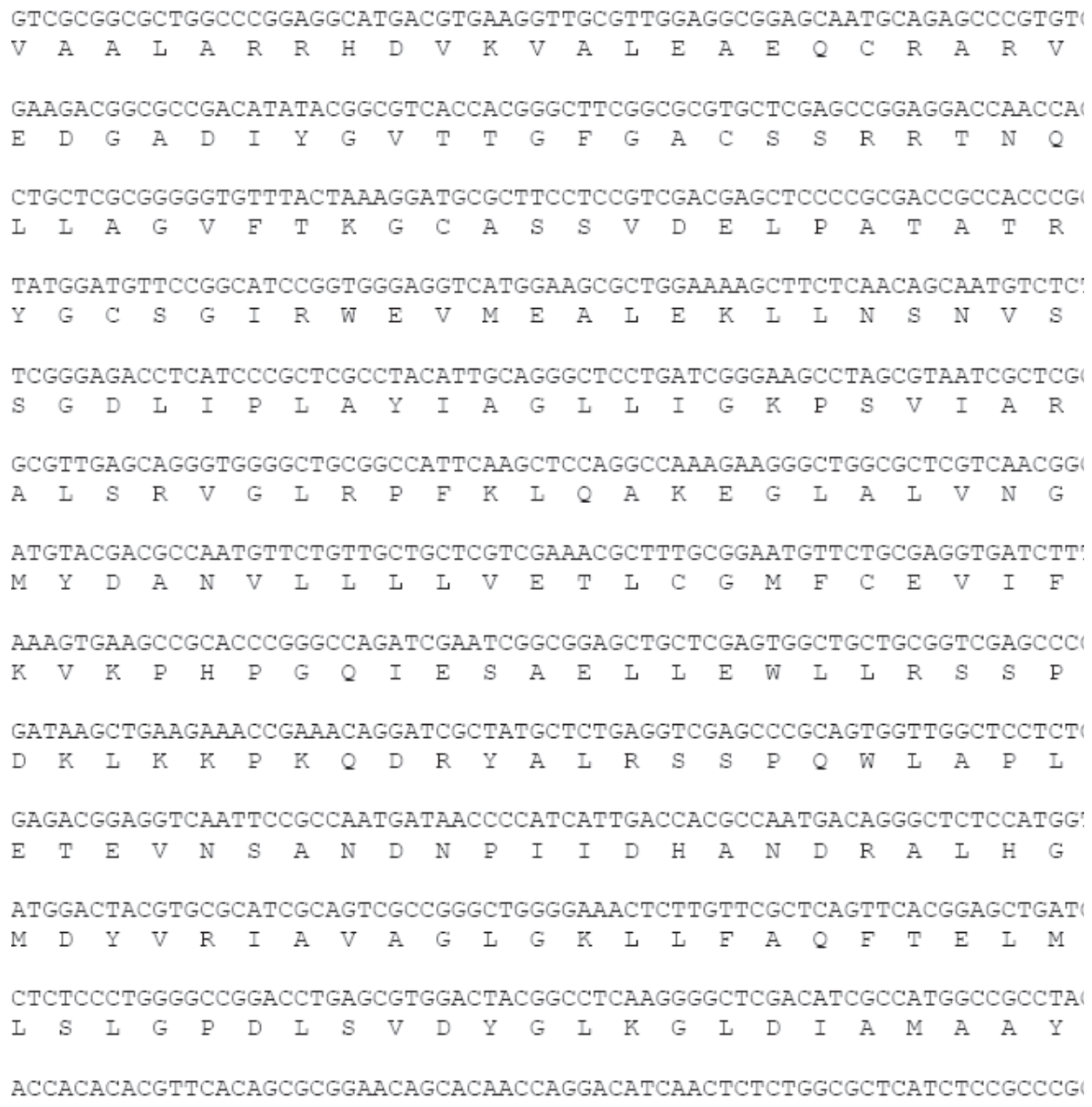




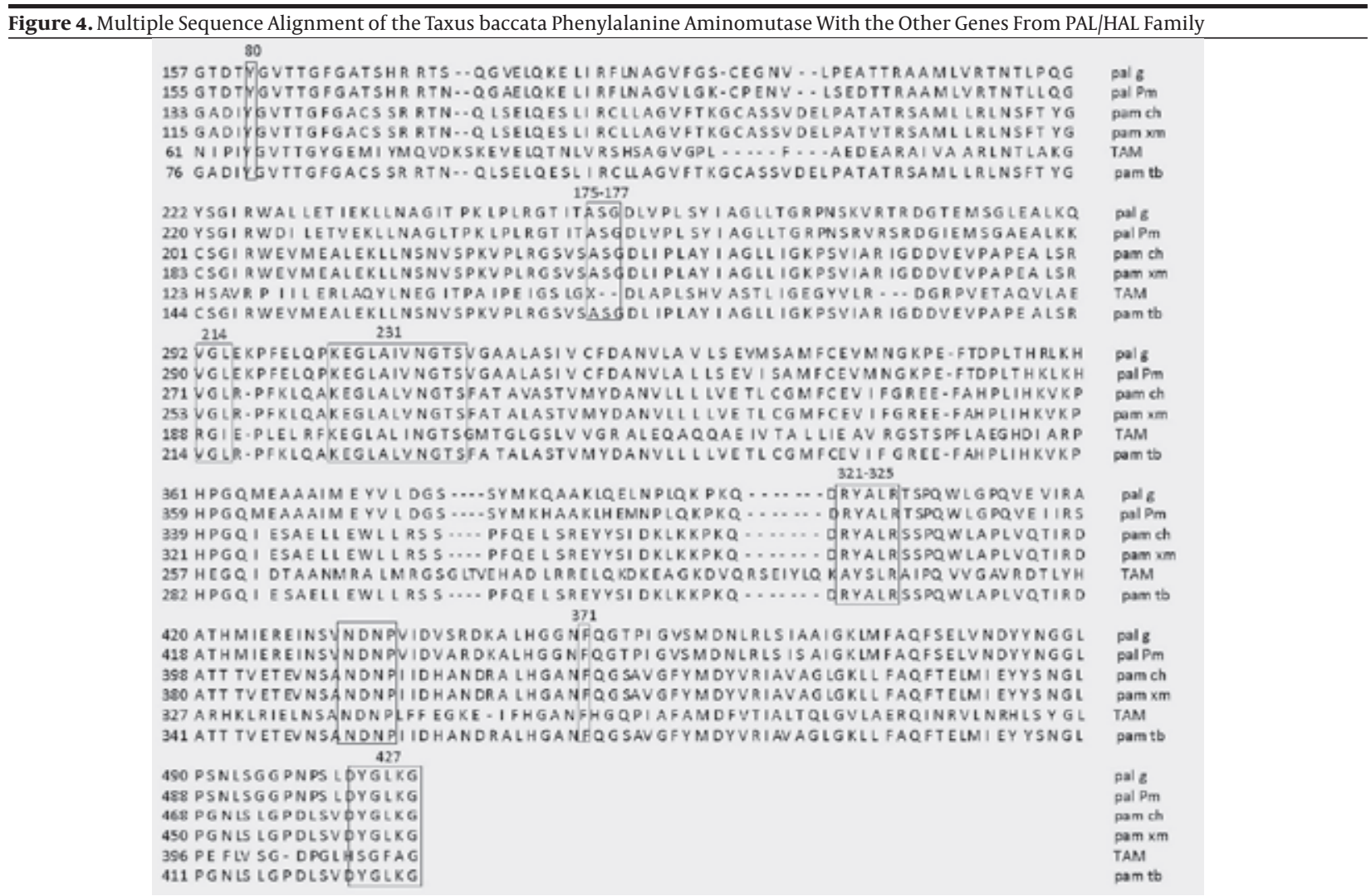

Pal g, Ginkgo biloba Phenylalanine Ammonialyase (ABU49842); pal pm, Pinus massoniana Phenylalanine Ammonialyase (ACS28225.2); pam ch, Taxus chinensis phenylalanine aminomutase (AAUO1183); pam xm, Taxus $x$ media phenylalanine aminomutase (AAUO1184); TAM, Tyrosine Aminomutase (ABY66005); pam tb, Taxus baccata phenylalanine aminomutase (ADA57703). Proposed active site (175-177) and binding sites (80, 214, 231, 322, 325, 371, 427 the numbering on the boxes based on pam tb sequence) showed in the boxes.

\subsection{Predicted Structure and Active Site of TbPAM}

Similarity searches using the BLAST algorithm indicated that the amino acid sequence of TbPAM is most similar(up to $98 \%$ ) to phenylalanine ammonia-lyase (PAL). Among the other aminomutases, Tyrosine Aminomutase (TAM) showed most similarity (70\%) to TbPAM with respect to the amino acid sequence. Based on PAL-derived TAL activity in monocots and fungi (10) and also high structure similarity between phenylalanine and tyrosine, it seems that the binding mechanism and mode of action could be similar in PAM and TAM. Based on this assumption and the scientific literature on the crystal structure of TAM (11), probable protein structure of TbPAM was predicted by using the phyre 2 server from the Centre for Bioinformatics Imperial College, London (Figure 5). The predicted structure of TbPAM adopts a predominantly $\alpha$-helical fold that is linked together by $\beta$-sheets. Such a structure could be essential for flexibility and plasticity of the enzyme and seems to be a functional requirement for substrate binding and catalysis. Data indicated that TbPAM has the same overall protein fold as tyrosine aminomutase (SgTAM) that was reported by Montavon and coworkers (11). Moreover, superposition of the theoretical structure of
TbPAM with TAM and PAL indicated significant homology where TbPAM contains a 4-methylidene-imidazole-5-one (MIO) cofactor. The MIO moiety formed in the active site of the enzyme by spontaneous (autocatalytic) cyclization and dehydration of an internal 175Ala-Ser-Gly177 tripeptide motif was similar to TAM and histidine/phenylalanine ammonia lyase (PAL/HAL) family (Figure 4). These similarities suggest a common mechanism in the vicinal interchange catalyzed by PAM and TAM, and also in the elimination of ammonia catalyzed by the related lyases. Furthermore, the amino acids involved in active site of SgTAM exactly conserved in TbPAM.

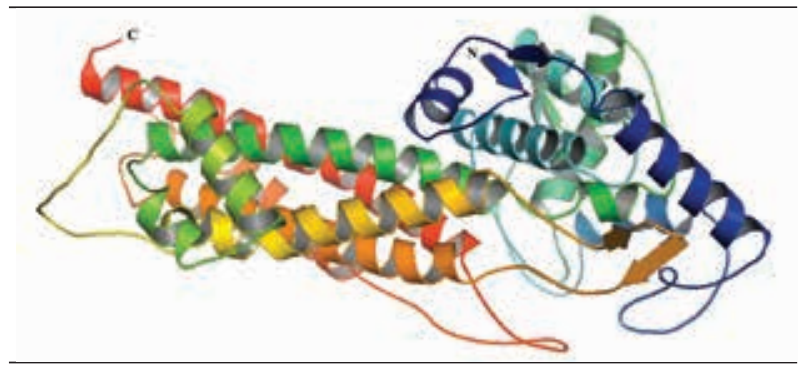

Figure 5. Predicted Structure of Phenylalanine Aminomutase from Taxus baccata L. 


\section{Discussion}

Study and manipulation of the enzymes involved in metabolic pathways are considered as one of the most efficient methods to improve productivity and also to alter pathways for production of novel compounds. Study of substrate specificity and enzymatic chemistry is an essential step towards this aim. Aminomutases that are classified as isomerases, catalyze the vicinal interchange of an amino group and hydrogen either via radical-dependent homolytic or ion-dependent heterolytic mechanisms. Taxus baccata PAM (TbPAM) catalyzes the conversion of $\alpha$-phenylalanine to $\beta$-phenylalanine; the first committed step in the biosynthesis of N-benzoyl phenylisoserinoyl side-chain of the anticancer drug,taxol. PAM revealed a multifunctional activity which catalyzes the isomerization of $2 S$ - $\alpha$-phenylalanine to $3 \mathrm{R}-\beta$-phenylalanine with retention of configuration at $\mathrm{C} 3$, reversely the conversion of both R- and S- $\beta$-phenylalanine to the same $2 S$ - $\alpha$ phenylalanine, and also the $\alpha, \beta$-elimination of ammonia from the $\alpha$-isomer to form trans-cinnamic acid (4). Recent mechanistic studies on the aminomutases (PAM and TAM) revealed that 4-hydroxycinnamic acid (in the case of TAM) and cinnamic acid (in the case of PAM) are intermediates in the aminomutase reaction that can be released from the active site of the enzyme. These observations indicate that TAM and PAM might exhibit ammonia lyase activity and suggest the possibility of using the second half of the aminomutase reaction to synthesize $\beta$-amino acids from cinnamates (12). Comparison of the amino acid sequence of TBPAM with PAL and TAM indicate that the enzyme also contain a signature Ala-Ser-Gly motif which rearranges auto-catalytically to the MIO moiety in the active site of the enzyme. Unlike the aminomutases that require external cofactors, PAM activity depends on a MIO-derived cofactor. Based on crystallographic experiments on the MIO-dependent tyrosine aminomutase, Christanson et al. (13) suggested that MIO reacts with the amino group of the substrate during the initial deamination step of the aminomutase reaction. It seems that the $\alpha$-amine of the substrate ( $\alpha$-phenylalanine) adds into the electrophilic moiety (MIO) via a conjugate addition that facilitates deprotonation of the $\beta$-hydrogen. In this condition, the carbon-nitrogen bond is broken and the MIO-NH2 bond and $\alpha, \beta$-unsaturated carboxylic acid are formed. Then the MIO-bound amine adds on at the $\beta$-position while these intermediates retain in the active site of the enzyme. Finally, the product ( $\beta$-phenylalanine) is ultimately released and the MIO group regenerated. Walker et al. (4) revealed that potassium cyanide and sodium borohydride both eliminate the function of the MIO in PAM similar to ammonia lyases. The result suggests that the MIO moiety is essential for the activity of PAM similar to TAM and PAL. Comparison of PAM from T. baccata to those previously acquired from other Taxus species revealed that these enzymes are very identical (99\%) at the amino acid level and they share, as expected, the signature active site motif 175Ala-Ser-Gly177. This may be related to the essential role of the enzyme in the taxol biosynthesis pathway. Similarity searches using the amino acid sequence of TbPAM indicated that the enzyme is most similar (up to 98\%) to phenylalanine ammonia-lyase (PAL) that catalyzes the first committed step in the phenylpropanoid biosynthetic pathway. PAM and PAL share several enzymatic properties specially the lack of cofactor requirements and monomeric molecular weight (14) that suggest a common mechanism in the action of these enzymes. The higher Km for PAM $(1100 \mu \mathrm{M})$ than PAL (29$260 \mu \mathrm{M}$ ) indicates that if the phenylpropanoid and the taxoid pathways were in direct competition for substrate then the phenylpropanoid pathway would be favored (14). More importantly, the high Km for PAM suggests that PAM may be a rate-limiting enzyme in the biosynthesis of taxol (5). Based on the points mentioned above and the relative abundance of some precursors such as baccatin III compared totaxol in the Taxus tissues, it seems that PAM catalyzes a rate limiting enzymatic step in taxol biosynthesis pathway and is considered as an important target for genetic engineering in yew. Over expressing the enzyme or genetic engineering to increase the affinity of the enzyme to the substrate in Taxus, may improve the biosynthesis of taxol. On the other hand, competition between PAM and PAL for $\alpha$-phenylalanine may be an important factor that decreases the efficiency of PAM.

\section{Acknowledgements}

Special thanks are extended to Dr. S.A. Hosseini Tafreshi, Dr. M.A. Malboobi, Dr. M.A. Asadollahi, and S. Kadkhodaei for their helpful suggestions.

\section{Authors' Contribution}

Abolghasem Abbasi Kajani: experimental procedures, preparing the manuscript, Mohammad Reza Mofid: bioinformatics analysis, the manuscript preparation, Khalil Alami Saeid: experimental procedures.

\section{Financial Disclosure}

This study was financially supported by Agricultural Biotechnology Research Institute, Central Region of Iran (ABRICI) and there is not any conflict of interest.

\section{Funding/Support}

Agricultural Research and Education Organization of Iran (AREO).

\section{References}

1. Frense D. Taxanes: perspectives for biotechnological production. Appl Microbiol Biotechnol. 2007;73(6):1233-40

2. Kai G, Miao Z, Qiu C, Zhang L, Zhao L, Li Z, et al. Molecular cloning and characterization of a taxadienol acetyl transferase cDNA from Taxus x media. Plant Sci. 2004;167(4):759-64

3. Abbasi Kejani A, Hosseini Tafreshi SA, Khayyam Nekouei SM, Mo- 
fid MR. Efficient isolation of high quality nucleic acids from different tissues of Taxus baccata L. Mol Biol Rep. 2010;37(2):797-800

4. Walker KD, Klettke K, Akiyama T, Croteau R. Cloning, heterologous expression, and characterization of a phenylalanine aminomutase involved in Taxol biosynthesis. I Biol Chem. 2004;279(52):53947-54

5. Steele CL, Chen Y, Dougherty BA, Li W, Hofstead S, Lam KS, et al. Purification, cloning, and functional expression of phenylalanine aminomutase: the first committed step in Taxol side-chain biosynthesis. Arch Biochem Biophys. 2005;438(1):1-10

6. Ghassempour A, Rezadoost H, Ahmadi M, Aboul-Enein HY Seasons Study of Four Important Taxanes and Purification of 10-Deacetylbaccatin III from the Needles of Taxus baccata L. by Two-Dimensional Liquid Chromatography. J Liquid Chromatography Related Tech. 2009;32(10):1434-47

7. Kajani AA, Mofid MR, Abolfazli K, Tafreshi SA. Encapsulated activated charcoal as a potent agent for improving taxane synthesis and recovery from cultures. Biotechnol Appl Biochem. 2010;56(2):71-6

8. Collins D, Mill RR, Moller M. Species separation of Taxus baccata, T. canadensis, and T. cuspidata (Taxaceae) and origins of their reputed hybrids inferred from RAPD and cPDNA data. Am J Bot.
2003;90(2):175-82

9. Wang YD, Yuan YJ, Wu JC. Induction studies of methyl jasmonate and salicylic acid on taxane production in suspension cultures of Taxus chinensis var. mairei. Biochem Eng J. 2004;19(3):259-65

10. Louie GV, Bowman ME, Moffitt MC, Baiga TJ, Moore BS, Noel JP. Structural determinants and modulation of substrate specificity in phenylalanine-tyrosine ammonia-lyases. Chem Biol. 2006;13(12):1327-38

11. Montavon TJ, Christianson CV, Festin GM, Shen B, Bruner SD. Design and characterization of mechanism-based inhibitors for the tyrosine aminomutase SgTAM. Bioorg Med Chem Lett. 2008;18(10):3099-102

12. Wu B, Szymanski W, Wietzes P, de Wildeman S, Poelarends GJ, Feringa BL, et al. Enzymatic synthesis of enantiopure alphaand beta-amino acids by phenylalanine aminomutase-catalysed amination of cinnamic acid derivatives. Chembiochem. 2009;10(2):338-44

13. Christianson CV, Montavon TJ, Festin GM, Cooke HA, Shen B, Bruner SD. The mechanism of MIO-based aminomutases in betaamino acid biosynthesis. J Am Chem Soc. 2007;129(51):15744-5

14. Dixon RA, Dey PM, Lamb CJ. Phytoalexins: enzymology and molecular biology. Adv Enzymol Relat Areas Mol Biol.1983;55:1-136 